\title{
Giant teratoma of anterior mediastinum in a 14-year-old girl as an example of potential diagnostic problems and errors
}

\author{
Przemysław Wolak ${ }^{1,2}$, Renata Skiba ${ }^{3}$, Wojciech Niedziela² \\ ${ }^{1}$ Department of Correct and Functional Anatomy, Institute of Nursing and Obstetrics, Faculty of Health Sciences, \\ Jan Kochanowski University, Kielce, Poland \\ Head of the Department: Prof. JKU Piotr L. Chłosta \\ ${ }^{2}$ Department of Pediatric Surgery, Urology and Traumatology, Voivodeship Specialist Pediatric Hospital, Kielce, Poland \\ Head of the Department: Dr. Piotr Stępien \\ ${ }^{3}$ Operating Theater of the Voivodeship Specialist Pediatric Hospital, Kielce, Poland \\ Head of the Theater: Dr. Wojciech Wąsacz
}

Key words: mediastinal teratoma, mediastinal tumor, fever of unknown etiology, exudative pleuritis.

\begin{abstract}
Teratomas are tumors originating from the three primary germ layers, most commonly located within gonads or in the sacral region. Chest locations are rare. Mediastinal teratoma showing no tumor-specific symptoms may be treated as exudative pneumonia. The goal of the article was to present a case encountered in our practice as a showcase of possible diagnostic problems and errors. Despite a thorough medical examination with additional exams (ultrasound scans of pleural cavities, chest X-ray and laboratory analyses), the diagnosis of a thoracic tumor was made only after chest computed tomography scan was performed following ineffective attempts at antibiotic therapy and pleural drainage. Following the diagnosis of mediastinal tumor, the patient was subjected to surgery. A giant teratoma (confirmed in histopathological examination) was removed upon left-sided thoracotomy. Following the procedure, lung expansion and patient recovery were observed. Computed tomography of the chest should be performed routinely upon encountering difficulties in the treatment of exudative pneumonia in children. In every case of pneumonia with pleural effusion in children, inflammatory mask of mediastinal tumors should be ruled out.
\end{abstract}

\section{Introduction}

Teratomas are tumors originating from the three primary germ layers. The term "teratoma" originates from the ancient Greek word for monster. It was first used by Virchow in 1869 to describe a tumor in the sacrocaudal region [1]. Teratomas are tumors derived from the ectoderm, endoderm and mesoderm, although they may also be formed of a single type of germ layer [2]. The most common locations include the sacrocaudal region (35-60\%) and gonads (ovaries and testicles), mediastinum (15\%), rarely stomach $(1 \%)$, retroperitoneal space $(5 \%)$ or intracranially $[1,2]$. Chest locations are rare. In particular, mediastinal teratoma showing no tumor-specific symptoms may be treated as exudative pneumonia. Teratomas can be classified as mature or immature (with the structures of yolk sac tumor or ca embryonale, respectively) $[1,3]$. In the case of immature teratomas, increase in the levels of tumor markers ( $\alpha$-fetal protein (AFP), human chorionic gonadotropin ( $\beta-\mathrm{HCG})$ ) may sometimes be observed $[1,4,5]$. Determination of the levels of these markers facilitates the diagnosis and assessment of the dynamics of the pathological process. In patients under follow-up observation after comple- tion of treatment, it may be the first indicator of cancer relapse. In most cases, teratomas are diagnosed immediately after birth or in infancy [3, 6-8].

The goal of the article was to present a case encountered in our practice as a showcase of possible diagnostic problems and errors. Despite a thorough medical examination with additional exams (ultrasound scans of pleural cavities, chest X-ray and laboratory analyses), the diagnosis of a thoracic tumor was made only after chest computed tomography (CT) scan was performed following ineffective attempts at antibiotic therapy and pleural drainage.

\section{Case report}

A 14-year-old girl was unsuccessfully treated for 7 days in an outpatient setting with an antibiotic (amoxicillin) for dyspnea, dry cough, fever reaching $39^{\circ} \mathrm{C}$, and pain in the chest and left subcostal region. Physical examination upon admission revealed dull percussion above the left lung and lack of audible respiratory murmur in the left chest. A classical X-ray of chest was taken in the P-A plane, revealing shading of the lower $2 / 3$ of the chest with relocation of the heart and mediastinum towards the right (Figure 1). 


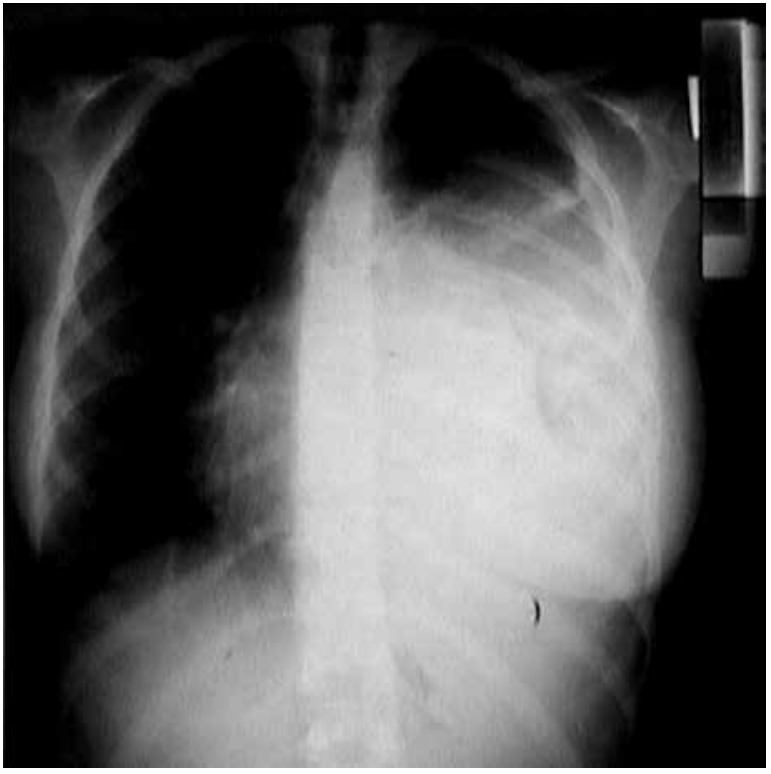

Figure 1. Preoperative X-ray of chest was taken in the P-A plane

The ultrasound scan of the pleura performed upon admission revealed a large amount of fluid (more than $1000 \mathrm{ml}$ ) in the left pleural cavity; fluid compartments were visible with part of the fluid being encysted; and the heart was relocated towards the right. Supportive examinations revealed no pathologies other than a positive C-reactive protein (CRP) result and elevated lactate dehydrogenase (LDH) level. Diagnosis of leftsided pneumonia was made and antibiotic treatment (cefuroxime) was initiated. The child was qualified for decompression of the left pleural cavity. The left pleural cavity was punctured to afford $750 \mathrm{ml}$ of xanthochromic fluid collected for general examination and cultures. The patient's health improved slightly as she experienced no fever and reported no dyspnea. The culture of the fluid collected from the chest revealed no growth of aerobic bacteria; general examination revealed no tumor cells. No tests were performed to screen for tuberculosis. In the follow-up ultrasound scan, the quantity of fluid within the left pleural cavity was measurable and amounted to $875 \mathrm{ml}$. The patient was transferred to the Department of Pediatric Surgery, Urology and Traumatology. Suction drainage was installed in the left pleural cavity, yielding $750 \mathrm{ml}$ of xanthochromic fluid. Follow-up ultrasound scan and X-ray examination revealed no improvement a suggestion was made to expand the diagnostic methods as the picture might correspond to cavernous necrosis in the course of pneumonia, infected cystic lung disease or hypertrophic lesion. Amikacin and fluconazole were added to the cefuroxime regime. A CT scan of the chest was performed on the $4^{\text {th }}$ day of hospitalization, revealing a cystiform, multicompartmental mass with calcifications and adipose tissue, causing

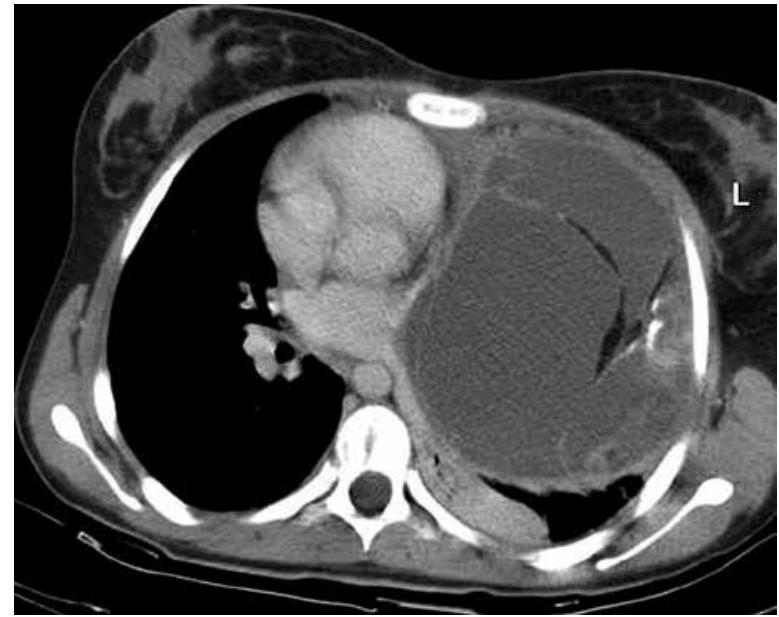

Figure 2. CT scan of the chest with tumor

relocation of mediastinal structures towards the right. The size of the lesion was ca. $170 \mathrm{~mm} \times 150 \mathrm{~mm} \times$ $105 \mathrm{~mm}$ (Figure 2). The left pleural cavity contained a fluid layer of 28-mm thickness; the drain was visualized. The image most likely corresponded to a hypertrophic lesion. The serum levels of tumor markers (AFP and $\beta$-HCG) were in the normal range. Bronchoscopic examination was performed (the left upper bronchium was compressed with the lumen reduced to ca. $30 \%$ compared to the contralateral structure). The patient was qualified for surgical treatment. Surgery was performed on the $10^{\text {th }}$ day after admission. Leftsided anterolateral thoracotomy was performed in the $5^{\text {th }}$ intercostal space, revealing a very large solid/cystic tumor, as well as a sunken and relocated left lung. The adhesions were removed, tumor volume was reduced by evacuation of fluid from the cystic space, the tumor was dissected from the diaphragm, pericardial sac and left lung; the lung was dissected using a linear stapler. The left lung was decompressed, filling the pleural cavity. The pleural cavity was evacuated using 2 drainage tubes extending through the $6^{\text {th }}$ intercostal space. The chest was closed in layers. The patient was transferred to the Department of Anesthesiology and Intensive Care. The post-operative recovery proceeded well. On the $3^{\text {rd }}$ day after the procedure, bronchoscopy was required with simultaneous aspiration of secretion from the left bronchium and its branches. Antibiotic therapy was continued. On the $5^{\text {th }}$ day after the surgery, the drainage tube was removed from the pleural cavity and the patient was transferred to the Department of Pediatric Surgery. The patient required respiratory rehabilitation. Macroscopic histopathological examination revealed a tumor sized $17 \mathrm{~cm} \times$ $\times 15 \mathrm{~cm} \times 10 \mathrm{~cm}$, with tooth buds, adipose tissue and hair visible in the cross-sections. The microscopic image corresponds to mature teratoma (teratoma maturum partim cysticum) (Figure 3). Tissues from three 


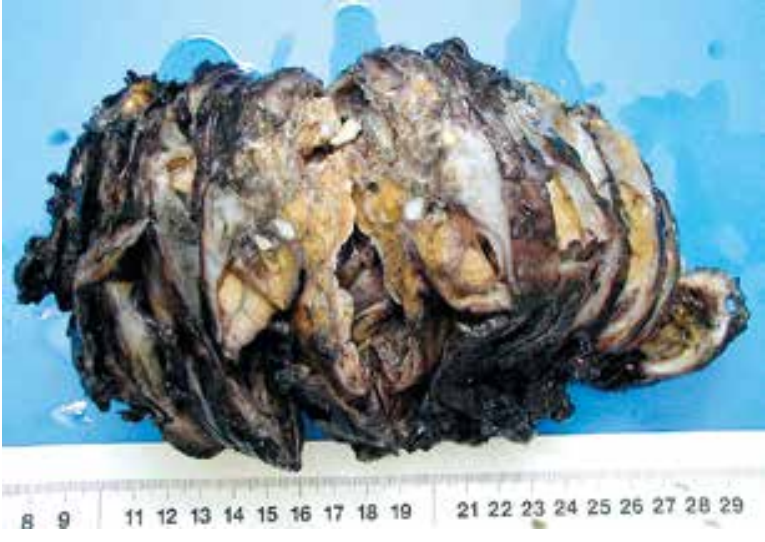

Figure 3. The macroscopic image of tumor

germ layers were identified within the tumor structure. The patient was discharged from the Department of Pediatric Surgery on the $10^{\text {th }}$ day after the surgical procedure, cardiovascularly and respiratorily stable, with the chest wound properly healed. The treatment was continued at the Department of Pneumology and Allergology, from which the patient was discharged home on the $15^{\text {th }}$ day after the surgery. The patient was under periodic observation at the Outpatient Clinic of Pediatric Surgery and the Outpatient Clinic of Pneumology, showing no complications of the surgical treatment for thoracic teratoma and no subsequent respiratory infection (Figure 4).

\section{Discussion}

In case of mediastinal location, teratoma may exert symptoms of respiratory inefficiency in neonates or infants, directly threatening the child's life [3, 6-8]. According to American authors, $42 \%$ of tumors are diagnosed prenatally [3]. Mediastinal teratomas are operated on mainly immediately after birth or during the first months of life [3, 6-9]. However, late diagnosis of thoracic teratomas may occur. A case similar to that of our patient was reported by Golash in a 20-year-old female patient [10]. The giant mass of the tumor (mature teratoma) located within the mediastinum, sized $27 \mathrm{~cm} \times 20 \mathrm{~cm} \times 11 \mathrm{~cm}$ and weighing $14 \mathrm{~kg}$, led to symptoms of respiratory disorders and difficulties swallowing due to the compression of the neighboring structures [10]. Another case was reported in Australia and pertained to an 18-year-old female patient with an immature teratoma sized $23 \mathrm{~cm} \times$ $17 \mathrm{~cm} \times 9 \mathrm{~cm}$, weighing $2005 \mathrm{~g}$ and located within the left pleural cavity [11]. Our case was associated with marked diagnostic difficulties. The patient was admitted for exudative pleuritis without any reservations from radiologists, pediatricians and surgeons with relation to the presence of pleural fluid that should have been drained. However, the lack of clinical improve-

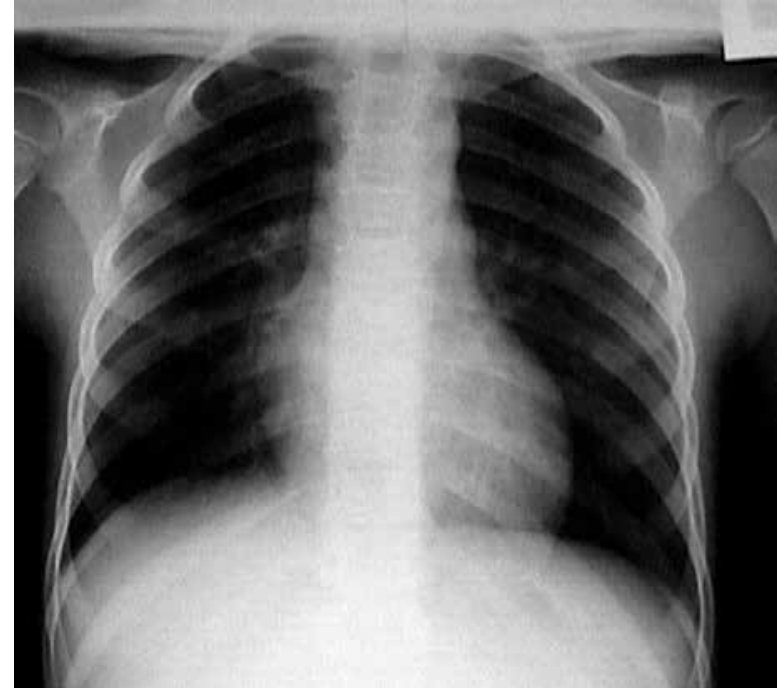

Figure 4. Postoperative X-ray of chest

ment, xanthochromia of the fluid that was expected to be purulent effusion and, most of all, lack of clear changes in ultrasound scans and X-ray examinations of the chest following evacuation of nearly $2 \mathrm{~L}$ of fluid from what we suspected to be the left pleural cavity, suggested otherwise. Expanding the diagnostic range by inclusion of the chest CT scan allowed us to make the correct diagnosis and qualify the patient for surgical treatment. Therefore, we propose the expansion of the diagnostic methods used in dubious cases by computed tomography or magnetic resonance scans, as also highlighted by other authors [2]. Classic X-rays may not visualize fragments of bones or teeth within the tumor outlines; in most cases, the picture is uncharacteristic [2]. Mature teratomas may grow over years, with patients adapting to the anatomical conditions changed by the growth of the tumor. The presence of the tumor may be disclosed only by accompanying infection or trauma with tumor bleeding. Surgical procedures usually allow for complete recovery, as was observed in our case as well.

\section{Conclusions}

Computed tomography of the chest should be performed routinely upon encountering difficulties in the diagnosis and treatment of exudative pneumonia in children. In every case of pneumonia with pleural effusion in children, inflammatory mask of chest tumors, including mediastinal tumors, should be ruled out.

\section{References}

1. Lakhoo K. Neonatal teratoma. Early Hum Develop 2010; 86: 643-647.

2. Kuniej T, Całka K, Jaźwiec P, et al. Radiological findings of large teratoma in anterior mediastinum. Pol J Radiol 2006; 71: 53-55. 
3. Isaacs H. Perinatal (fetal and neonatal) germ cell tumors. J Ped Surg 2004; 39: 1003-1013.

4. Chen CK, Chang YL, Jou ST, et al. Treatment of mediastinal immature teratoma in a child with precocious puberty and Klinefelter's syndrome. Ann Thorac Surg 2006; 82: 1906-1908.

5. Afifi $\mathrm{H}$, Bosl G, Burt M. Mediastinal growing teratoma syndrome. Ann Torac Surg 1997; 64: 359-362.

6. Martino F, Avila L, Encinas J, et al. Teratomas of the neck and mediastinum in children. Pediatr Surg Int 2006; 22: 627-634.

7. Kuroiwa M, Suzuki N, Takahashi A, et al. Life-threatening mediastinal teratoma in a neonate. Pediatr Surg Int 2001; 17: 235-238.

8. Halkiewicz F, Gumprecht K, Kostowska-Kapłon U, et al Potworniak dojrzały w śródpiersiu jako przyczyna ostrej niewydolności oddechowej u 5-miesięcznego niemowlęcia. Pneumol Alerg Pol 2004; 72: 343.

9. Dzielicki J, Gregorek J, Dobrowolska B. Guzy śródpiersia jako przyczyna duszności u dzieci. Pediatr Pol 1997; 72: 321-323.

10. Golash V. A giant anterior mediastinal teratoma presenting as orthopnea et dysphagia in an adult. J Thorac Cardiovasc Surg 2005; 130: 612-613.

11. Mc Leod NP, Vallely MP, Mathur MN. Massive immature mediastinal teratoma extending into the left pleural cavity. Heart Lung Circulation 2005; 14: 45-47.

\section{Address for correspondence:}

Przemysław Wolak

Institute of Nursing and Obstetrics

Department of Health Sciences

Jan Kochanowski University

al. IX Wieków Kielc 19, 25-317 Kielce, Poland

Phone: +48 501525549

E-mail: przemyslaw.wolak@ujk.edu.pl 